\title{
Trachoma and ocular Chlamydia trachomatis rates in children in trachoma-endemic communities enrolled for at least three years in the Tanzania National Trachoma Control Programme
}

\author{
H. MKOCHA*, B. MUNOZ and S. WEST \\ Kongwa Trachoma Project, Kongwa, P.O. Box 124, Kongwa, Tanzania \\ Dana Center for Preventive Ophthalmology, Johns Hopkins University, Baltimore MD, USA
}

\begin{abstract}
Trachoma, a blinding eye disease caused by repeated and prolonged infection with Chlamydia trachomatis, is a significant public health problem for sub-Saharan Africa. Tanzania has had a National Trachoma Task Force since 1999, working on trachoma control in endemic districts. The objective of this study was twofold: first, to determine the current status of infection and clinical trachoma in these districts in Tanzania, and second, to determine if a combination of clinical signs could be used as a surrogate for infection. We conducted a survey for trachoma and infection with C. trachomatis in 75 villages in eight districts of Kongwa, Kilosa, Mpwapwa, Bahi, Kondoa, Manyoni, Monduli and Iramba in Tanzania, which have previously been shown to be endemic. In each village, a random sample of households, and of children within households, was taken for examination. Trachoma was graded using the World Health Organization system, which we expanded, and a swab taken to determine presence of infection. The rates of trachoma ranged from $0 \%$ in Iramba District to $15.17 \%$ in Monduli District, with large variation in villages within districts. Infection rates were generally lower than trachoma rates, as expected, and most districts had villages with no infection. A combination of clinical signs of trachoma in children, when absent, showed very high specificity for identifying villages with no infection. We conclude that these signs might be useful for monitoring absence of infection in villages, and that districts with trachoma prevalence between $10 \%$ and $15 \%$ should have village level rapid surveys to avoid unnecessary mass treatment.
\end{abstract}

Key words: prevalence, trachoma, Chlamydia, Tanzania

\section{Introduction}

Trachoma is the leading infectious cause of blindness worldwide, affecting an estimated 40.6 million people of whom 8.2 million have trichiasis (Mariotti et al., 2009; Resnikoff et al., 2004). Mariotti et al estimated that in Tanzania, 1.22 million persons suffer from trachoma and 214 thousand from trichiasis in endemic areas (Mariotti et al., 2009). This chronic conjunctivitis, caused by repeated episodes of infection with Chlamydia trachomatis, afflicts the most impoverished communities on earth. Because of its absence in developed countries, trachoma was largely forgotten as a public health issue until a new antibiotic donation program coupled with renewed focus by the World Health Organization (WHO) rekindled interest in eradicating blinding trachoma.

The WHO Alliance for the Global Elimination of Blinding Trachoma by the year 2020 (GET 2020) has endorsed a multifaceted trachoma control program for countries endemic for trachoma (WHA, 2003). There is sound public health reason for this focus: the economic costs of trachoma in endemic countries are estimated at an annual productivity loss of $\$ 2.9$ billion, based on loss of vision (Frick et al., 2003). The prevalent cases of visual loss are responsible for 39 million lifetime Disability -Adjusted Life Years (DALYS). These impacts are likely to be under-estimates, as trichiasis (the potentially blinding sequelae of years of ocular infection), even without vision loss, is associated with disability (Frick et al., 2001).

In communities endemic for trachoma, the pool of active inflammatory trachoma resides in the young children who may have persistent signs of active trachoma as result of repeated or persistent infections (West et al., 1991; West et al., 1996). In hyperendemic areas, active disease prevalence in pre-school children can be as high as high as $60 \%$ to $90 \%$ (West et al., 1991, 1996). Using quantitative PCR in Tanzania, we have shown $90 \%$ of infection within the community resides in pre-school children (West et al., 1991, 1996; Solomon et al., 2003; West et al., 2005). Multiple infections over time and/

* Correspondence: Harran Mkocha; E-mail: harry_mkocha2@yahoo.com 
or prolonged, severe infection are followed by evidence of scarring of the conjunctiva. As early as childhood and early adult hood, the scarring may be clearly evident; scarring can lead to trichiasis (Munoz et al., 1999).

Use of antibiotics to treat infection within the community is one of the cornerstones of the WHO endorsed "SAFE" strategy for countries implementing trachoma control programmes (WHA,2003).Countryprogrammesaremanaging once yearly treatments, with varying success at reaching target coverage of $80 \%$. Tanzania for several years targeted $75 \%$ coverage and took two rounds of mass treatment to achieve such coverage (West et al., 2000, 2001, 2002). Even more frequent treatment has been suggested to be more effective in reducing infection over time in hyperendemic countries, specifically biannual treatment, based on mathematical modeling (Lietman et al., 1999).

Tanzania has had an active trachoma control programme since 1999, implementing the full SAFE strategy in endemic districts. In this project, we undertook prevalence surveys for trachoma and ocular chlamydia infection during 2007-2008 in villages in endemic districts around Tanzania to determine the current state of trachoma. We report the prevalence within these districts.

\section{Materials and Methods}

\section{Study areas}

This study was carried out in eight districts endemic of trachoma in Tanzania. They included Kilosa in Morogoro Region, Kongwa, Mpwapwa and Bahi in Dodoma Region, Manyoni and Iramba in Singida Region and Monduli in Arusha Region.

The Tanzanian National Trachoma Control programme managers have been enrolling villages within the trachoma endemic districts, and enrolling new districts, each year since 1999. The program strives to implement the full SAFE strategy, training trichiasis surgeons for each district, and providing flip charts and radio programmes on face washing and importance of clean environments for each district. The program is also the recipient of azithromycin donation, to be given as mass treatment in a single dose, $20 \mathrm{mg} / \mathrm{kg}$, to those aged one year and older. Mass treatment is scheduled every year. The programme has grown from twelve villages in each of six districts in 1999 to 32 districts with close to 600 villages in 2008.
The villages that were eligible for this study were those that had baseline data on trachoma rates prior to the program and for whom the National Trachoma Control Programme had data on coverage of within the community of azithromycin. In addition, they had to have at least three rounds of mass treatment. We stratified the villages in the study area by number of mass treatments in the village to be sure we were not over-representing villages that had many rounds, or villages that had few. Seventy-five villages were randomly selected, representing 8 districts.

\section{Sentinel children}

We surveyed a sentinel sample of 100 to 120 children aged five and under, randomly selected in each village. Selection of children was done as follows: after discussion with the village elders and approval obtained to conduct the study in their village, we asked each of the mtaa leaders to list each of their tencell leaders. We then asked each of the ten cell leaders to list the head of household for the ten to twenty households in their cells. We listed all households in the village by ten cell listing and numbered them sequentially. From this list we will select the households from which the preschool child will be selected. We aimed for 100120 children per village; past response rates to surveys have been $78 \%$, but the lowest in these villages at our baseline survey was around $70 \%$; therefore to account for refusals, we aimed for 143 households. The number of households on the list would be divided by 143 to arrive at the sampling interval, " $\mathrm{n}$ ". With a random start on the list every "nth" household would be selected to be in the study. From past experience, about $70 \%$ of households in these villages have at least one child aged five years and under. If a household did not contain a child aged five years or less, the household would be ineligible, and the next household on the list, previously not selected, would be selected as replacement. In the Iramba district, as we suspected the rates would be low, we sampled 150 children.

Within the household, we randomly selected only one child aged five years or less from the eligible household. We know that trachoma clusters within households, and by selecting only one child per household we avoided that level of clustering. Once the child was selected, a notice was sent to the family, inviting them to attend the survey where details of the examination were provided, and informed, written consent was obtained. 


\section{Data Collection}

One senior grader (HM) performed all assessments of trachoma in the field, and took an ocular photograph of every child for quality control purposes. In addition, an ocular swab of the left upper eyelid of each child was taken following strict procedures to avoid field contamination. A laboratory assistant wore sterile gloves, and flipped the eyelid. He was the only one to actually touch the child around the eyes (the mother is often enlisted to hold the child). The senior grader, also wearing sterile gloves, removed the sterile Dacron swab by grabbing it 2/3 of the way down the shaft, not touching the swab itself. He then rubbed the swab across the eyelid three times, twirling the swab as it goes across the tarsal plate, then inserted it into the vial, careful not to touch the edge or inside of the vial, and re-capped the vial. All personnel changed gloves between each child. At the end of every tenth participant, we have swabbed the hands of the person taking the swab for a control.

The vial was placed in a cold box in the field and was transferred each evening to were denatured, and sent for detection using probe-coated microwell plates. Detection is accomplished by measuring the optical density at $\mathrm{A}_{450^{\circ}}$. The assay result for the negative controls should be less than $0.2 \mathrm{~A}_{450^{\prime}}$ and the assay result for the positive controls should be 0.8 or greater for ocular specimens for a valid run.

We tested separately for amplification of both the target plasmid DNA and the mastermix internal control samples to determine if PCR was inhibited. Samples whose values in valid runs were $\geq 0.8 \mathrm{~A}_{450}$ were counted as positive, and samples less than $0.2 \mathrm{~A}_{450}$ were negative. Samples for which the result were equivocal $(\geq 0.2,<0.8)$ were tested again; if equivocal twice, they were left as equivocal and called not positive in the analyses as no run equaled 0.8 or greater.

For this study, we have expanded the World Health Organization simplified grading scheme to accommodate milder disease, and more severe signs (Thylefors, 1987). This expanded scheme permits us to collapse to the simplified system (Table 1).

Table 1: Expanded classification of Trachoma, based on World Health Organization Simplified Grading Scheme

\begin{tabular}{|c|c|c|c|c|}
\hline Sign & None (0) & Mild (1) & $\begin{array}{l}\text { WHO Grading Scheme } \\
\text { Minimum Definition (2) }\end{array}$ & Severe (3) \\
\hline $\begin{array}{l}\text { Follicular } \\
\text { trachoma (TF) }\end{array}$ & $\begin{array}{l}0=\text { no follicles size } \\
0.5 \mathrm{~mm}\end{array}$ & $\begin{array}{l}\text { 1=1-4 follicles size } 0.5 \\
\mathrm{~mm}\end{array}$ & $\begin{array}{l}2=5-10 \text { follicles size } 0.5 \\
\mathrm{~mm}\end{array}$ & $\begin{array}{l}3=>10 \text { follicles size } \\
0.5 \mathrm{~mm}\end{array}$ \\
\hline $\begin{array}{l}\text { Intense } \\
\text { Trachoma (TI) }\end{array}$ & $\begin{array}{l}0=\text { no obscuring of } \\
\text { deep tarsal vessels }\end{array}$ & $\begin{array}{l}\text { 1= inflammatory } \\
\text { thickening and } \leq 50 \% \\
\text { of deep tarsal vessels } \\
\text { obscured }\end{array}$ & $\begin{array}{l}2=\text { inflammatory } \\
\text { thickening and }>50 \% \\
\text { of deep tarsal vessels } \\
\text { obscured }\end{array}$ & $\begin{array}{l}3=\text { no vessels visible } \\
\text { due to inflammatory } \\
\text { thickening }\end{array}$ \\
\hline
\end{tabular}

a freezer, and stored until shipped to Johns Hopkins International Chlamydia Laboratory, frozen, for processing. The specimens were processed according to strict protocol, outlined in the manufacturer's kit directions. The Roche Amplicor C. trachomatis qualitative PCR assay from Roche Molecular Systems was used. Procedures are summarized as follows: Each swab was eluted by vortexing in Amplicor $\mathrm{CT} / \mathrm{NG}$ lysis buffer in polypropylene tubes, and then Amplicor specimen diluent was added. Using a known positive sample in the laboratory, positive and negative $C$. trachomatis (CT) processing controls were created; two CT+ and two CT- processing controls were run with each batch of specimens. The Working master mix was created and the specimens prepared prior to amplification. The specimens and controls were placed in the thermal cycler for amplification. Once completed, the specimens
With this scheme, the WHO grade of TF equals grades 2 and 3 for follicular trachoma, and TI equals grades 2 and 3 for intense trachoma. We assessed the reliability of this grading scheme using 59 photographs of persons randomly selected from one village in Tanzania. The photographs were read twice, on different days, by one experienced trachoma grader who was masked to previous grades when re-grading the set. The sample of photographs contained $27 \%$ TF and 19\% TI, so the sample has a reasonable distribution of persons with disease. The unweighted kappa for intra-observer agreement using the expanded scheme was 0.65 for TF and 0.78 for TI.

\section{Data analysis}

The prevalence of ocular chlamydia infection, $\mathrm{TF}, \mathrm{TI}$, and the corresponding exact 95\% Confidence Intervals (CI) assuming a binomial 
distribution are presented at community level. The average prevalence and 95\% CI was estimated for each district. To examine the association between infection and severity of the trachoma clinical sign, the proportion of children positive for infection is presented for all possible combinations of the severity of TF and TI

\section{Results}

A total of 75 villages and 8342 children were examined. The average village prevalence of active trachoma (TF or $\mathrm{TI}$ ) when each village enrolled in the National Trachoma Control program at the start was $50.3 \%$ (range $=17$ $79 \%$ ). The average prevalence of TF (with or without TI) and TI (with or without TF) in each district in our survey is shown in Table 2. Active trachoma (TF or TI) is also shown. The rates of TF ranged from $0 \%$ in Iramba (District 12) to 15.17 Monduli (District 10). However, the data show a large variation in trachoma and infection rates among the villages within the districts. In Kongwa district, seven villages had TF rates less than $10 \%$, yet two villages still had trachoma rates greater than $20 \%$. Because the overall prevalence rate of TF is estimated at greater than $10 \%$, the entire district (following WHO guidelines) would be mass treated.

The average prevalence of infection in each district is shown in Table 2. The infection rates varied from 0 in Iramba to $8.5 \%$ in Monduli, similar to the trachoma rates. As expected, infection rates generally were lower than trachoma rates in each district. However, there were some villages where the infection rate was actually higher than the rate of active trachoma, notably in Kilosa (district 2) where the rates of trachoma were low.

If we exclude Iramba where there was probably no trachoma at baseline, then only 4 of $48(8.3 \%)$ villages with any TI had no infection. Only one village of the 17 where TI was greater than $5 \%$ had no infection. While TI presence seems to be a good marker of infection, the absence of TI does not necessarily indicate the absence of infection. In the 23 villages with no TI (again excluding those from Iramba) only 4 also had no infection (17\%). However, of the five villages with no TI where the infection rates were greater than $5 \%$, three of the five were in Kilosa district where trachoma was likely reemerging and perhaps were sub clinical cases.

Table 2: Prevalence of Trachoma and C. trachomatis infection by district and village

\begin{tabular}{|c|c|c|c|c|c|}
\hline District & Village & \# children & $\begin{array}{l}\text { Prevalence of TF2 or } \\
\text { worse (with or without } \\
\text { TI) }(95 \% \mathrm{CI})\end{array}$ & $\begin{array}{l}\text { Prevalence of TI } 2 \text { or worse } \\
\text { (with or without TF2) } \\
(95 \% \text { CI) }\end{array}$ & $\begin{array}{l}\text { Prevalence of infection } \\
(95 \% \mathrm{CI})\end{array}$ \\
\hline \multirow[t]{13}{*}{ Kongwa } & 0101 & 112 & $5.36(1.99-11.30)$ & $5.36(1.99-11.30)$ & $1.79(0.22-6.30)$ \\
\hline & 0102 & 106 & $3.77(1.04-9.38)$ & $0.94(0.02-5.14)$ & $2.83(0.59-8.05)$ \\
\hline & 0103 & 106 & $4.72(0.68-8.75)$ & $1.89(0.02-6.65)$ & $5.66(2.11-11.91)$ \\
\hline & 0104 & 106 & 8.49 (3.96-15.51) & $2.83(0.06-8.05)$ & $10.38(5.30-17.81)$ \\
\hline & 0105 & 106 & $10.38(5.30-17.8)$ & $2.83(0.06-8.05)$ & $6.60(2.70-13.13)$ \\
\hline & 0106 & 113 & $14.16(8.32-21.97)$ & $12.39(6.94-19.91)$ & $6.19(2.53-12.35)$ \\
\hline & 0107 & 113 & $9.73(4.96-16.75)$ & $1.77(0.02-6.25)$ & $6.19(2.53-12.35)$ \\
\hline & 0108 & 113 & $12.39(6.94-19.91)$ & $3.54(0.97-8.82)$ & $9.73(4.96-16.75)$ \\
\hline & 0109 & 113 & $23.89(16.37-32.86)$ & $12.39(6.94-19.91)$ & $23.01(15.61-31.87)$ \\
\hline & 0113 & 113 & $8.85(4.33-15.67)$ & $2.65(0.05-7.56)$ & 7.08 (3.11-13.47) \\
\hline & 0114 & 128 & $22.66(15.73-30.89)$ & $7.81(3.81-13.90)$ & $10.16(5.52-16.74)$ \\
\hline & 0115 & 106 & $12.26(6.69-20.06)$ & $6.60(2.70-13.13)$ & $4.72(1.55-10.77)$ \\
\hline & 0116 & 120 & $7.50(3.49-13.76)$ & $1.67(0.02-589)$ & $4.17(1.37-9.46)$ \\
\hline Total & & 13 villages & $11.09(7.33-14.84)$ & $4.82(2.45-7.19)$ & $7.58(4.35-10.08)$ \\
\hline \multirow[t]{10}{*}{ Kilosa } & 0201 & 106 & $3.77(1.04-9.38)$ & $0.00(0.00-3.42)$ & $1.89(0.23-6.65)$ \\
\hline & 0202 & 106 & $2.83(0.59-8.05)$ & $0.00(0.00-3.42)$ & $5.67(2.11-11.91)$ \\
\hline & 0203 & 106 & $6.60(2.70-13.13)$ & $0.94(0.02-5.14)$ & $7.55(3.31-14.53)$ \\
\hline & 0204 & 106 & 3.77 (1.04-9.38) & $0.94(0.02-5.14)$ & $13.21(7.41-21.17)$ \\
\hline & 0205 & 113 & $5.31(1.97-11.20)$ & $0.88(0.02-4.83)$ & $7.96(3.71-15.58)$ \\
\hline & 0206 & 113 & $5.31(1.97-11.20)$ & $0.00(0.00-3.21)$ & $9.73(4.96-16.75)$ \\
\hline & 0213 & 120 & $8.33(4.07-14.79)$ & $1.67(0.20-5.89)$ & $5.83(2.38-11.65)$ \\
\hline & 0214 & 120 & $6.67(2.92-12.71)$ & $0.00(0.00-3.03)$ & $5.83(2.38-11.65)$ \\
\hline & 0215 & 120 & $5.83(2.38-11.65)$ & $1.67(0.20-5.89)$ & $12.50(7.17-19.78)$ \\
\hline & 0219 & 106 & $1.89(0.23-6.65)$ & $0.00(0.00-3.42)$ & $0.00(0.00-3.42)$ \\
\hline
\end{tabular}




\begin{tabular}{|c|c|c|c|c|c|}
\hline & 0220 & 106 & $0.94(0.02-5.14)$ & $0.00(0.00-3.42)$ & $0.94(0.02-5.14)$ \\
\hline & 0221 & 106 & $2.83(0.60-8.05)$ & $0.00(0.00-3.42)$ & 3.77 (1.04-9.38) \\
\hline & 0222 & 106 & $1.89(0.02-6.65)$ & $0.94(0.02-5.14)$ & $0.00(0.00-3.42)$ \\
\hline & 0223 & 106 & $0.00(0.00-3.42)$ & $0.00(0.00-3.42)$ & $0.00(0.00-3.42)$ \\
\hline & 0224 & 106 & $6.60(2.70-13.13)$ & $2.83(0.60-8.05)$ & $4.72(1.55-10.67)$ \\
\hline Total & & 15 villages & $4.17(2.82-5.52)$ & $0.66(0.02-1.14)$ & $4.31(2.90-7.71)$ \\
\hline \multirow{9}{*}{ Mpwapwa } & 0301 & 106 & $9.43(462-16.67)$ & $0.00(0.00-3.42)$ & $10.38(5.30-17.81)$ \\
\hline & 0302 & 106 & $17.92(11.2-26.57)$ & 7.55 (3.31-14.3) & $9.43(4.62-16.67)$ \\
\hline & 0303 & 106 & $17.92(11.2-26.57)$ & $1.89(0.23-6.65)$ & $11.32(5.99-18.94)$ \\
\hline & 0304 & 120 & $9.17(4.67-15.81)$ & $8.33(4.07-14.79)$ & $2.50(0.52-7.13)$ \\
\hline & 0305 & 120 & $8.33(4.07-14.79)$ & $8.33(4.07-14.79)$ & $6.67(2.92-12.71)$ \\
\hline & 0306 & 120 & $26.67(19.01-35.51)$ & $15.00(9.14-22.67)$ & $21.67(14.67-30.11)$ \\
\hline & 0313 & 120 & $15.83(9.81-23.62)$ & $0.00(0.00-3.03)$ & $2.50(0.52-7.13)$ \\
\hline & 0314 & 120 & $5.00(1.86-10.57)$ & $0.00(0.00-3.03)$ & $0.83(0.02-4.56)$ \\
\hline & 0315 & 120 & $28.33(20.49-37.28)$ & $6.67(2.92-12.71)$ & $25.00(17.55-33.73)$ \\
\hline Total & & 9 villages & $15.40(9.09-21.71)$ & $5.31(1.32-9.30)$ & $10.03(3.54-16.53)$ \\
\hline \multirow[t]{5}{*}{ Bahi } & 0401 & 113 & $10.62(5.61-17.82)$ & $3.54(0.97-8.82)$ & 3.63 (1.00-9.05) \\
\hline & 0402 & 113 & $2.65(0.55-7.56)$ & $0.88(0.02-4.83)$ & $3.54(0.97-8.82)$ \\
\hline & 0403 & 91 & 4.40 (1.21-10.87) & $0.00(0.00-3.97)$ & $0.00(0.00-3.97)$ \\
\hline & 0404 & 106 & $8.49(3.96-15.51)$ & $2.83(0.59-8.05)$ & $2.83(0.59-8.05)$ \\
\hline & 0405 & 106 & 8.49 (3.96-15.51) & $1.89(0.23-6.65)$ & $5.66(2.11-11.91)$ \\
\hline Total & & 5 villages & $6.93(2.85-11.01)$ & $1.83(0.05-3.60)$ & $3.13(0.69-5.57)$ \\
\hline \multirow[t]{9}{*}{ Kondoa } & 0501 & 94 & $8.51(3.75-16.08)$ & $3.19(0.66-9.04)$ & $2.13(0.26-7.48)$ \\
\hline & 0502 & 113 & 8.85 (4.33-15.67) & 3.54 (0.97- 8.82) & $1.77(0.22-6.25)$ \\
\hline & 0503 & 133 & $9.02(4.75-15.23)$ & $2.26(0.47-6.45)$ & $0.75(0.02-4.12)$ \\
\hline & 0504 & 113 & 12.39 (6.94-19.91) & $10.62(5.61-17.82)$ & $5.31(1.97-11.20)$ \\
\hline & 0505 & 133 & 7.52 (3.66-13.39) & $4.51(1.67-9.56)$ & $3.76(1.23-8.56)$ \\
\hline & 0506 & 113 & $7.08(3.11-13.47)$ & $2.65(0.55-7.56)$ & $0.00(0.00-3.21)$ \\
\hline & 0513 & 106 & $10.38(5.30-17.81)$ & $4.72(1.55-10.67)$ & $0.94(0.02-5.14)$ \\
\hline & 0514 & 106 & $5.66(2.11-11.91)$ & $0.00(0.00-3.42)$ & $0.00(0.00-3.42)$ \\
\hline & 0515 & 106 & $6.60(2.70-13.13)$ & $2.83(0.59-8.05)$ & $3.77(0.01-7.46)$ \\
\hline Total & & 9 villages & $8.45(6.87-10.02)$ & $3.81(1.58-6.04)$ & $2.05(0.61-3.48)$ \\
\hline \multirow[t]{14}{*}{ Manyoni } & 0601 & 113 & 15.04 (9.01-22.99) & $0.00(0.00-3.21)$ & $2.65(0.55-7.56)$ \\
\hline & 0602 & 106 & $9.43(4.62-16.67)$ & $0.94(0.02-5.14)$ & $0.00(0.00-3.42)$ \\
\hline & 0603 & 106 & $17.92(11.15-26.57)$ & $2.83(0.59-8.05)$ & 7.55 (3.31-14.33) \\
\hline & 0604 & 114 & 8.77 (4.29-15.54) & $0.88(0.02-2.479)$ & $1.77(0.22-6.25)$ \\
\hline & 0605 & 113 & 13.27 (7.62-20.95) & $0.00(0.00-3.21)$ & $1.77(0.22-6.25)$ \\
\hline & 0606 & 106 & $9.43(4.62-16.67)$ & $0.00(0.00-3.42)$ & $2.83(0.59-8.05)$ \\
\hline & 0614 & 120 & $10.00(5.27-16.82)$ & $0.00(0.00-3.03)$ & $1.67(0.02-5.89)$ \\
\hline & 0615 & 120 & $8.33(4.07-14.79)$ & $0.00(0.00-3.03)$ & $00.0(0.00-3.03)$ \\
\hline & 0619 & 106 & $21.70(14.28-30.76)$ & $2.83(0.85-8.05)$ & 3.77 (1.04-9.38) \\
\hline & 0620 & 106 & 11.32 (5.99-18.94) & $0.94(0.02-5.14)$ & $0.94(0.02-5.14)$ \\
\hline & 0621 & 106 & $5.66(2.11-11.91)$ & $0.00(0.00-3.42)$ & $2.83(0.59-8.05)$ \\
\hline & 0622 & 106 & $6.60(2.70-13.13)$ & $0.00(0.00-3.42)$ & $0.95(0.02-5.19)$ \\
\hline & 0623 & 106 & $4.72(1.55-10.77)$ & $0.00(0.00-3.42)$ & $2.83(0.59-8.05)$ \\
\hline & 0624 & 106 & $21.70(14.28-30.76)$ & $5.66(2.11-11.91)$ & 7.55 (3.31-14.33) \\
\hline Total & & 14 villages & $11.71(8.52-14.90)$ & $0.94(0.00-1.92)$ & $2.65(1.30-4.01)$ \\
\hline \multirow[t]{6}{*}{ Monduli } & 1002 & 100 & $17.00(10.23-25.82)$ & $16.00(9.43-24.68)$ & $18.00(11.03-26.95)$ \\
\hline & 1004 & 100 & $20.00(12.67-29.18)$ & $9.00(4.20-16.40)$ & $11.00(5.62-18.83)$ \\
\hline & 1005 & 100 & $10.00(4.90-17.62)$ & $3.00(0.62-8.52)$ & $1.01(0.03-5.50)$ \\
\hline & 1006 & 100 & $7.00(2.86-13.89)$ & $1.00(0.03-5.45)$ & $0.00(0.00-3.62)$ \\
\hline & 1007 & 100 & $9.00(4.20-16.40)$ & $5.00(1.64-11.28)$ & $8.00(3.52-15.16)$ \\
\hline & 1009 & 100 & $28.00(19.48-37.87)$ & $17.00(10.23-25.82)$ & $9.00(4.20-16.40)$ \\
\hline Total & & 6 villages & $15.17(6.73-23.60)$ & $8.50(1.42-15.57)$ & $7.83(0.83-14.84)$ \\
\hline \multirow[t]{4}{*}{ Iramba } & 1201 & 73 & $0.00(0.00-4.93)$ & $0.00(0.00-4.93)$ & $0.00(0.00-4.93)$ \\
\hline & 1202 & 150 & $0.00(0.00-2.43)$ & $0.00(0.00-2.43)$ & $0.00(0.00-2.43)$ \\
\hline & 1203 & 150 & $0.00(0.00-2.43)$ & $0.00(0.00-2.43)$ & $0.00(0.00-2.43)$ \\
\hline & 1204 & 150 & $0.00(0.00-2.43)$ & $0.00(0.00-2.43)$ & $0.00(0.00-2.43)$ \\
\hline Total & & 6 villages & 0 & 0 & 0 \\
\hline
\end{tabular}


The association between the prevalence of infection and the clinical signs of trachoma is shown in Table 3. While severe TI was a consistent indicator of infection-over $50 \%$ of children were infected if they had this sign, regardless of the TF status, it was very rare; only 55 of 7811 children had this sign $(0.7 \%)$. Children with trachoma who met WHO criteria of TF and TI also had infection rates greater than $50 \%$, as did children with severe TF (ten or more follicles) who had at least some evidence of inflammation.

\section{Discussion}

In general, there has been clear benefit to Tanzania of the National Trachoma Control program, and the use of mass treatment of trachoma with donated azithromycin in the districts under study. Although the average prevalence of active trachoma at the start was $50 \%$ in children aged seven years and under in these villages (the pre-school age group chosen for study at the start of the programme), it is

Table 3: Prevalence of Infection with C. trachomatis within combinations of the clinical signs of trachoma

\begin{tabular}{llllll}
\hline TF & None & Mild & $\begin{array}{l}\text { WHO Grading } \\
\text { Scheme }\end{array}$ & Severe & All \\
\hline None & $(119 / 5822)$ & $(23 / 299)$ & $(2 / 33)$ & $(10 / 20)$ & $(154 / 6174)$ \\
& 2.04 & 7.7 & 6.1 & 50.0 & 2.5 \\
Mild & $(40 / 680)$ & $(46 / 278)$ & $(11 / 41)$ & $(8 / 15)$ & $(105 / 1014)$ \\
& 5.9 & 16.6 & 26.8 & 53.3 & 10.4 \\
WHO Grading & $(27 / 249)$ & $(26 / 109)$ & $(24 / 36) 66.7$ & $(5 / 9) 55.6$ & $(82 / 404)$ \\
Scheme & & 23.8 & & & 20.3 \\
Severe & $(15 / 89)$ & $(45 / 83)$ & $(22 / 37)$ & $(6 / 11)$ & $(88 / 220)$ \\
& 16.9 & 54.2 & 59.5 & 54.6 & 40.0 \\
All & $(201 / 6840)$ & $(140 / 769)$ & $(59 / 147)$ & $(29 / 55)$ & \\
& 2.9 & 18.2 & 40.1 & 52.7 & \\
\hline
\end{tabular}

Table 4 compares the combination to infection rates in the other disease categories and to those with no signs of trachoma. Children with one of the "high risk" combination signs have an infection rate of $56.8 \%$, while those with other signs of trachoma have a rate of $10 \%$, compared to no signs of trachoma at all, with an infection rate of $2 \%$. The sensitivity of this high risk combination of signs is low, but the specificity (for absence of infection)is high, $96 \%$. now $12 \%$ in those aged five years and under, the age group at highest risk that we chose to study. Tanzania must now consider moving some of its districts into another phase of control, and these data pose some significant issues for such a move.

The WHO recognizes so-called "implementation units" where the trachoma control program for the villages is organized.

Table 4: Comparison of Infection in "High Risk" trachoma signs*, low risk trachoma signs, and with no signs of trachoma

\begin{tabular}{lllll}
\hline Signs of Trachoma & Infection Present & Infection Absent & Total & Rate of infection \\
\hline "High Risk" signs & 120 & 91 & 211 & $56.8 \%$ \\
"Low Risk" signs & 190 & 1588 & 1778 & $10.7 \%$ \\
No trachoma & 119 & 5703 & 5822 & $2.0 \%$ \\
Total & 429 & 7382 & 7811 & \\
\hline
\end{tabular}

* High risk: Severe TI (grade 3), or severe TF (grade 3)with grade 1 or 2 TI, or TF grade 2 AND TI grade 1

Low risk: TF grade one with no severe TI, or TF grade 2 with no TI or only TI grade 1, Severe TF with no TI, and TI grade 1 or 2 with no TF 
In Tanzania, this is typically the district level, where eye care coordinators organize surgery, provide drugs for mass treatment, and implement health education programmes. WHO has recommended that when the district has trachoma prevalence greater than $10 \%$ in children aged under ten years, the entire district should be mass treated with azithromycin. (Such an indication means that the rates in children aged five and under will be even higher, because they typically have the highest prevalence rates in the community (West et al., 1991). However, there is currently some concern for the production levels of azithromycin and whether more judicious use in countries may become necessary.

Our data suggest a different approach to $\mathrm{WHO}$ guidelines. In this regard, for example, Kongwa district has overall TF rates in the age group five years and under greater than $10 \%$, whichsuggests the districtshould bemass treating every village. With 66 villages (excluding the district town) the population to be mass treated is estimated at 198,000 persons, and likely over 200,000 . However, our data also show the huge variation in villages, where of the 13 villages, 7 or $54 \%$ would clearly qualify to stop mass treatment with rates of TF less than $10 \%$. Two of the villages ( 2 of 13 or $15.4 \%$ ) would even qualify to stop all antibiotic treatment, with trachoma rates less than $5 \%$. The rest may still qualify for mass treatment (unless the rate in the expanded age group under ten years is less than $10 \%$ ). A similar situation occurs in Manyoni District, where the overall rates of $11.7 \%$ suggests mass treating the entire district, but we again observe large individual variation among villages. Our data suggest that when district prevalence rates are just above $10 \%$ (say between $10 \%$ and $15 \%)$ rapid assessment village by village in the district may be appropriate to identify which villages need mass treatment, which may get just targeted treatment and which may be stopped for treatment altogether. Such district level variation is not unique, and has been reported in a recent study in Kenya (Karimurio et al., 2006)

It is also worth noting that none of the villages in Kongwa, and only two in Manyoni, had a complete absence of infection in the children sampled. While in general, infection rates were low when trachoma rates were low, it was not altogether absent. Thus, the possibility of re-emergence of trachoma is possible if the village has not changed the environmental conditions that favor trachoma transmission. We have shown that after two mass treatments, when infection was low, infection and trachoma did reemerge in a formerly hyper-endemic community (West et al., 2007). Thus, if mass treatment is stopped, some surveillance following cessation is indicated.

Re-emergence of infection may be an issue in Kilosa, where infection rates in some villages are greater than trachoma rates. This finding is unlikely due to differences in assessment of trachoma between villages, because one grader did all the assessments, and we monitored grading for drift over time. The laboratory assessment of infection was done masked to clinical disease state and vice versa. We monitored the field team for evidence of field contamination, with no evidence. Rates of trachoma are very low in Kilosa, with an average trachoma prevalence of $4.17 \%$ and average infection rate of $4.92 \%$. This district would likely be eligible to stop mass treatment for all its villages under WHO guidelines, although stopping all villages would be of concern as clearly some villages have infection rates in children greater than $10 \%$, although disease rates are low. Some surveillance for re-emergence in this district is clearly indicated.

Iramba had no infection or trachoma. As this district had had mass treatment with less than $80 \%$ coverage for only three years, it is unlikely there would be no trace of infection or clinical disease. It is more likely that the baseline survey had overestimated the trachoma rates in that district.

We correlated the various clinical signs of disease with infection rates, to see if improvement in detection of infection was possible. Other research has shown that TI is better correlated with infection than TF (West et al., 1991; West et al., 2005). We also found a good correlation between villages which had any TI and the presence of infection. However, if TI is not present, it does not necessarily mean there is not chlamydia infection, although largely the infection rate was $5 \%$ or less. We have identified a combination of signs, representing about $11 \%$ of those with any sign of trachoma, and $25 \%$ of those with WHO criteria for trachoma, where infection rates exceed $50 \%$ if the sign is present. The sensitivity of this combination of these signs is low, but the specificity is very high, $96 \%$. Thus, if the combination of these signs is not seen in the sample of children in the village, the likelihood of infection in the village is very low, regardless if other signs are seen. Such a screening tool, using clinical signs to determine if there may be residual infection in the village, needs validation but might be useful until a rapid diagnostic, point of care, test for infection is available.

In summary, we have shown variation within district in trachoma rates in the villages, which would be of little importance if the district 
is hyper-endemic for trachoma as virtually all of the villages have trachoma rates greater than $10 \%$. However, if the district prevalence is greater than $10 \%$ and less than $15 \%$, it is important to consider rapid assessment of all villages to avoid mass treating villages that no longer need intensive treatment. At the same time, districts whose average prevalence falls below $10 \%$ should have some surveillance system in place to ensure re-emergence does not occur. A rapid point-of-care test for Chlamydia that could be used for surveillance would be ideal for such surveillance, but in the absence, using a combination of trachoma signs that include severe trachoma grades might be useful.

\section{Acknowledgements}

This project was supported by a grant from the National Institutes of Health, National Eye Institute EY16429. We are grateful to the villages and children of Tanzania for their cooperation.

Received 20 March 2009

Revised 19 June 2009

Accepted 23 June 2009

\section{References}

Frick, K.D., Basilion, E.V., Hanson, C.L. \& Colchero, M.A. (2003) Estimating the burden and economic impact of trachomatous visual loss. Ophthalmic Epidemiology 10, 121-132.

Frick, K.D., Melia, B.M., Buhrmann, R.R., \& West, S.K. (2001) Trichiasis and disability in a trachoma-endemic area of Tanzania. Archives of Ophthalmology 119,1839-1844.

Karimurio, J., Gichanqi, M., Ilako, D.R., Adala, H.S. \& Kilima, P. (2006) Prevalence of trachoma in six districts of Kenya. East African Medical Journal, 83, 63-68.

Lietman, T., Porco, T., Dawson, C., \& Blower, S. (1999) Global elimination of trachoma: how frequently should we administer mass chemotherapy? Nature Medicine 5, 572-576.

Mariotti, S.P., Pascolini, D., \& Rose-Nussbaumer, J. (2009) Trachoma: global magnitude of a preventable cause of blindness. British Journal of Ophthalmology, 93, 563-568.

Munoz, B., Bobo, L., Mkocha, H., Lynch, M., Hsieh, Y.H., \& West, S.K. (1999) Incidence of trichiasis in a cohort of women with and without scarring. International Journal of Epidemiology, 28, 1167-1171.

Resnikoff, S., Pascolini, D., Etya'ale, D., Kocur, I., Pararajasegaram, R., Pokharel, G.P. \& Mariotti S.P. (2004) Global data on visual impairment in the year 2002. Bulletin of the World Health Organization 82, 844-851.
Solomon, A.W., Holland, M.J., Burton, M.J., West, S.K., Alexander, N.D., Aguirre, A., Massae, P.A., Mkocha, H., Munoz, B., Johnson, G.J., Peeling, R.W., Bailey, R.L., Foster, A. \& Mabey, D.C. (2003) Strategies for control of trachoma: observational study with quantitative PCR. Lancet, 362 (9379),198-204.

Thylefors, B., Dawson, C.R., Jones, B.R., West, S.K. \& Taylor, H.R. (1987) A simple system for the assessment of trachoma and its complications. Bulletin of the World Health Organization, 65, 477-483.

West, E.S., Munoz, B., Mkocha, H., Holland, M.J., Aguirre, A., Solomon, A.W., Bailey R.L., Foster, A., Mabey, D.C. \& West, S.K. (2005) Mass treatment and the effect on the load of Chlamydia trachomatis infection in a trachoma-hyperendemic community. Investigative Ophthalmology and Visual Sciences 46, 83-87.

West, S.K., Munoz, B., Lynch, M., Kayongoya, A., Mmbaga, B.B.O. \& Taylor, H.R.. (1996) Risk factors for constant, severe trachoma in pre-school children in Kongwa, Tanzania. American Journal of Epidemiology 143, 73-78.

West, S.K., Munoz, B., Mkocha, H., Gaydos, C. \& Quinn, T. (2007) Trachoma and ocular Chlamydia trachomatis were not eliminated three years after two rounds of mass treatment in a trachoma hyperendemic village. Investigative Ophthalmology and Visual Sciences 48, 1492-1497.

West, S.K., Munoz, B., Mkocha, H., Holland, M.J., Aguirre, A., Solomon, A.W. Foster, A., Bailey, R.L., \& Mabey, D.C. (2005) Infection with Chlamydia trachomatis after mass treatment of a trachoma hyperendemic community in Tanzania: a longitudinal study. Lancet, 366,12961300.

West,S.K.,Munoz,B.,Mkocha,H.,Ngirwamungu, E. \& Kilima, P. (2000) Annual Report to the International Trachoma Initiative: One Year Evaluation.

West,S.K.,Munoz,B.,Mkocha,H.,Ngirwamungu, E., \& Kilima P. (2001) Annual Report to the International Trachoma Initiative: Two Year Evaluation.

West,S.K.,Munoz,B.,Mkocha,H.,Ngirwamungu, E. \& Kilima, P. (2002) Annual Report to the International Trachoma Initiative: Three Year Evaluation.

West, S.K., Munoz, B., Turner, V.M., Mmbaga, B.B.O. \& Taylor, H.R. (1991) The epidemiology of trachoma in Central Tanzania. International Journal of Epidemiology 20,1088-1092. 